\title{
HYBRID SOLUTION APPROACHES FOR TACKLING MEMBRANE PROTEINS
}

\author{
H.D.T. Mertens ${ }^{\mathrm{a}}$ and D.I. Svergun ${ }^{\mathrm{a}}$ \\ ${ }^{a}$ European Molecular Biology Laboratory, Notkesstr. 85, 22607 Hamburg, Germany \\ ${ }^{b}$ Victor Chang Cardiac Research Institute, 405 Liverpool Street, 2010 Sydney, NSW, Australia
}

Membrane proteins are essential for homeostasis, compartmentalization of external and internal chemical reactions and an array of important biological functions. Evolution has led to the development of membrane embedded amphiphilic assemblies of proteins that operate as machinery regulating the flow of essential molecules, in and out of the cell [1].

Lipid embedded integral membrane proteins are difficult to study using traditional highresolution methods in structural biology. However, exploiting method complementarity by using

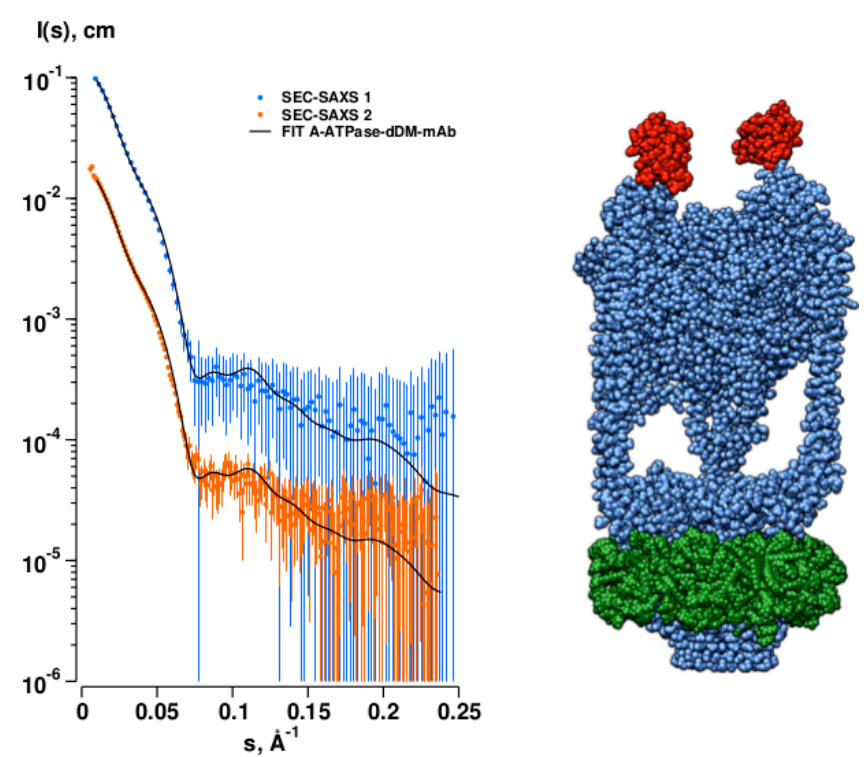

Figure 1. SAXS data for A-ATPase and hybrid model hybrid approaches covering a wide resolution range yields significant promise. Combining bioinformatics, X-ray crystallography, nuclear magnetic resonance, cryo-electron microscopy, mass spectrometry and small-angle X-ray and neutron scattering can yield results that together generate important insights into the mechanism of action of large lipid soluble complexes. An overview will be presented of the current state of the art of hybrid methods with a focus on the complementarity of SAXS and SANS. Methods developed are expected to guide future efforts to capture snapshots of biological processes

Difficulties encountered with the data acquisition in a detergent environment will be presented and the practical implications of studying such multi-contrast systems discussed. The development of effective screening procedures for these increasingly important protein systems in lipidic environments, combined with accurate modelling procedures will be outlined for these challenging systems.

\section{References}

[1] Stewart, A.G., E.M. Laming, M. Sobti, and D. Stock. "Rotary ATPases-dynamic molecular machines." Current opinion in structural biology 25 (2014): 40-48.

Submitting Author: hmertens@embl-hamburg.de 